Part of Journal of Research of the National Bureau of Standards, Volume 19, July 1937

\title{
EXTENSION OF NORMAL-INCIDENCE IONOSPHERE MEASUREMENTS TO OBLIQUE-INCIDENCE RADIO TRANSMISSION
}

\section{By Newbern Smith}

\section{ABSTRACT}

A simple, rapid graphical method is given for obtaining skip distances and limiting frequencies for radio waves, from normal-incidence measurements. The applications of the method are outlined.

\section{CONTENTS}

I. Introduction

II. Theory

III. Transmission curves

IV. Effect of the earth's curvature

V. Approximate method of calculation _...

\section{INTRODUCTION}

It is possible to interpret certain aspects of long-distance radiotransmission conditions in terms of normal-incidence ionosphere measurements of virtual heights and critical frequencies. It is the purpose of this note to outline a simple, rapid graphical method of obtaining, from these normal-incidence measurements, skip-distance, maximum usable frequency over a given path, and virtual heights of reflection of waves incident obliquely upon the ionosphere. This method has been used at the National Bureau of Standards since the beginning of 1936 for interpreting radio-transmission data in terms of the regular ionosphere measurements. More recently it has been used in preparing the data for the weekly broadcasts of radio-transmission conditions, in interpreting low-layer ionosphere transmission, and in supplementing local ionosphere measurements by data obtained from observations of long-distance transmission. Part of this work was presented as a paper at the 1936 joint meeting of the Institute of Radio Engineers and International Scientific Radio Union at Washington. ${ }^{1}$

\section{THEORY}

Breit and Tuve's theorem ${ }^{2}$ states that in the absence of dissipation a wave traversing a trajectory in the ionosphere undergoes the same group retardation as a wave traversing with the velocity of light a triangular path of the same base as the actual path and with the same angle of incidence on the ionosphere.

\footnotetext{
1 N. Smith, S. S. Kirby, and T. R. Gilliland. Recent correlations between the ionosphere and high-frequency radio transmission. Paper before joint meeting of IRE and URSI, May 1, 1936. To be published in J. Research NBS and Proc. Inst. Radio Engrs.

2 G. Breit and M. A. Tuve, Phys. Rev. 28, 554 (1926).
} 
The process of refraction and reflection of a radio wave in the ionosphere depends on the variation of the refractive index $\mu$ of the medium with the ionization density $N$. From the elementary theory of propagation of electromagnetic waves in a medium of varying refractive index, it follows that the wave will penetrate the medium only until the refractive index is reduced to the value $\sin \phi_{0}$, where $\phi_{0}$ is the angle of incidence of the wave on the ionosphere (see fig. 1), that is, to the level $z_{0}$. For the normal incidence case, this means that reflection takes place at the level where $\mu=0$.

Breit and Tuve's theorem states implicitly that if reflection takes place at a level $z_{0}$, the virtual height $z_{v}$, or height of the equivalent triangular path, is constant regardless of the value of $\phi_{0}$. If we assume the validity of this theorem, therefore, it follows that the virtual height measured at normal incidence for a frequency $f$ that makes $\mu=0$ for some value of $N$, will also be the virtual height attained in transmission over a distance by a wave of a different frequency $f^{\prime}$, such that $\mu=\sin \phi_{0}$ for this same value of $N$.

Instead of using the ionization density $N$, we shall use a quantity $f_{0}$, of the nature of a frequency and defined as

$$
f_{0}=\sqrt{\frac{N e^{2}}{\pi m}}
$$

Using this quantity and the quantities $f_{T}$ and $f_{L}$, which represent, respectively, the frequencies of precession of electrons in the components of the earth's magnetic field transverse to and along the direction of phase propagation, we may write the Appleton-Hartree formula ${ }^{3}$ for the refractive index of a nondissipative ionized medium in the presence of the earth's magnetic field, for a radio wave of frequency $f^{\prime}$ :

$$
\mu^{2}=1-\frac{f_{0}{ }^{2}}{f^{\prime 2}-\frac{f^{\prime 2} f_{T^{2}}}{2\left(f^{\prime 2}-f_{0}^{2}\right)} \pm \sqrt{\left[\frac{f^{\prime 2} f^{2} T}{2\left(f^{\prime 2}-f_{0}^{2}\right)}\right]^{2}+f^{\prime 2} f_{L}{ }^{2}}}
$$

The refractive index vanishes for $f_{0}=f^{\prime}$ and $f_{0}^{2}=f^{\prime}\left(f^{\prime} \pm f_{H}\right)$ where $f_{H}{ }^{2}=f_{T}{ }^{2}+f_{L}{ }^{2}$. The component of the wave reflected at normal incidence from the level where $f_{0}=f^{\prime}$ is known as the ordinary component, and the one reflected from the level where $f_{0}{ }^{2}=f^{\prime}\left(f^{\prime} \pm f_{H}\right)$ is known as the extraordinary component. Usually the extraordinary component is reflected mostly from only one of these levels (the one where $f_{0}^{2}=f^{\prime}\left(f^{\prime}-f_{H}\right)$ for $f^{\prime}>f_{H}$ and the one where $f_{0}^{2}=f^{\prime}\left(f^{\prime}+f_{H}\right)$ for $\left.f^{\prime}<f_{H}\right)$.

By using the Appleton-Hartree formula with $f_{T}$ and $f_{L}$ evaluated at the top of the wave path, where the direction of propagation is horizontal, and setting $\mu=\sin \phi_{0}$, we may obtain a set of values of $f_{0}$ and $\phi_{0}$, for a given value of $f^{\prime}$, representing transmission conditions at a given frequency. It is possible to express $f_{0}$ in terms of a frequency $f$ of a wave at normal-incidence for either the ordinary or extraordinary components by using the above relations, and it is possible to express $\phi_{0}$ in terms of virtual height $z_{0}$ and distance of transmission $D$ (fig. 1), by a purely geometrical relation. We can thus obtain a set of values of normal-incidence equivalent frequencies

3 E. V. Appleton, J. Inst. Elec. Engrs. (London) 71, 642 (1932). 
$f$ and virtual heights $z_{v}$, representing radio-transmission conditions for a given frequency $f^{\prime}$ over a given distance $D$.

\section{TRANSMISSION CURVES}

If, in normal incidence measurements, a wave of frequency $f$ is returned from a virtual height equal to the $z_{0}$ computed for a given distance and transmission frequency $f^{\prime}$, the wave of frequency $f^{\prime}$ will be reflected from a region of ionization density equal to that for which $\mu=0$ for the frequency $f$ and its equivalent triangular ray path will correspond to this virtual height, by Breit and 'Tuve's theorem. This point of reflection may be readily determined as the intersection of a normal-incidence frequency-virtual-height curve of the type obtained in multifrequency ionosphere experiments, with a curve plotted from the set of values of $f$ and $z_{0}$ obtained for a given $f^{\prime}$ and $D$. This latter curve may be called a transmission curve for the given frequency and distance.

This development is valid both for ordinary and extraordinary rays. For the ordinary ray, the transmission curve is, at least in

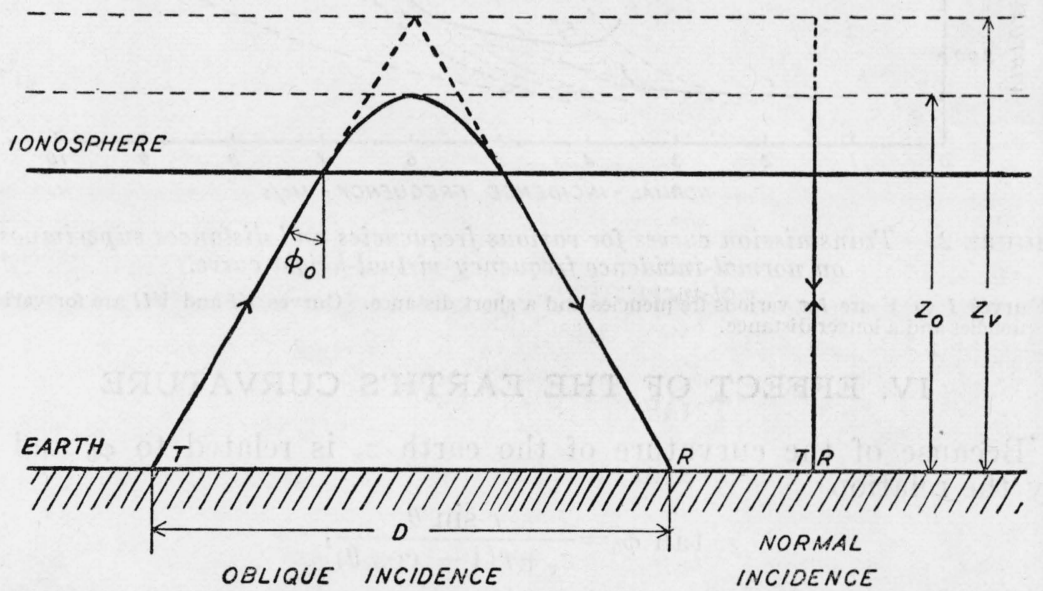

FIGURE 1.-Normal-incidence and oblique-incidence ionosphere transmission

$T$ is the transmitter, and $R$ is the receiver. The solid line is the actual path taken by the waves and the dotted line shows the equivalent triangular path.

these latitudes, very nearly a "secant-law" curve, i. e., $f=f^{\prime} / \mathrm{sec} \phi_{0}$. In computing the transmission curve for the ordinary ray the virtual height for the oblique-incidence case is that for the ordinary ray component of frequency $f$ at normal incidence, and corresponds to the frequency $f_{0}$. This ionization density is used in computing $\phi_{0}$, and the transmission curve is applied to the ordinary ray frequencyvirtual-height curve. The extraordinary component can be treated similarly.

The solid curve in figure 2 is a typical frequency-virtual-height curve for the ordinary component, showing $E$ and $F_{2}$ layers. Superimposed on it are the dotted transmission curves for various frequencies and distances. Curves $I, I I, I I I, I V$, and $V$ are for increasing frequencies and for a fairly short distance. For the frequency corresponding to $I$, transmission is by way of $E$ layer. For curve $I I$ 
the given distance is within the skip zone for $E$-layer transmission, but is transmitted by $F_{2}$ layer. Curve III shows transmission by two paths (high and low virtual heights), as reported in various papers. ${ }^{4}$ Curve $I V$ corresponds to the maximum possible frequency that can be used over the given distance. As the $F_{2}$ critical frequency varies, transmission has been observed to begin and fail quite abruptly at this point. For curve $V$ the frequency is too high and the given distance is within the skip zone for any ordinary-ray transmission. Curves $V I$ and $V I I$ are for a longer distance, and show a condition where $E$-layer transmission takes place at higher frequencies than $F$-layer transmission. Curve $V I I$ shows the skip effect again.

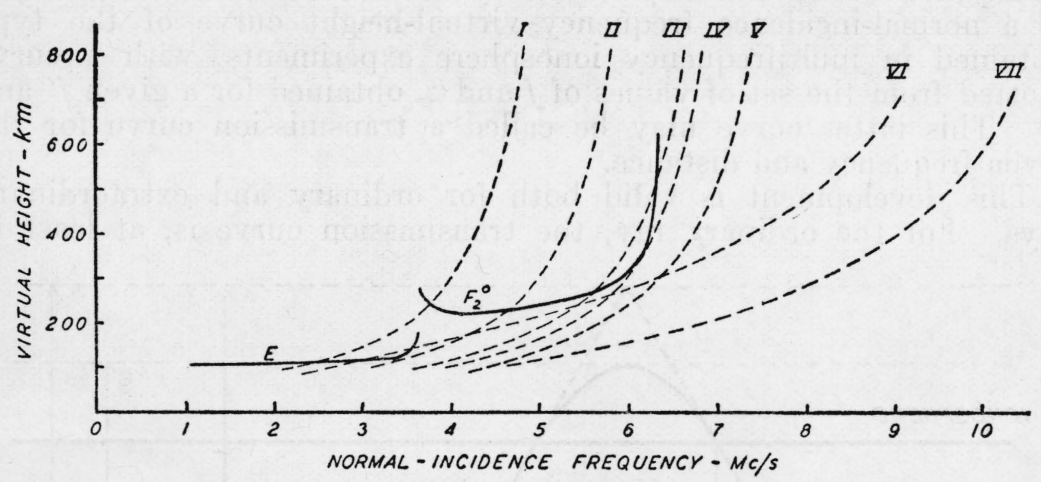

FIGURE 2.-Transmission curves for various frequencies and distances superimposed on normal-incidence frequency-virtual-height curve.

Curves $I$ to $V$ are for various frequencies and a short distance. Curves $V I$ and $V I I$ are for various frequencies and a longer distance.

\section{EFFECT OF THE EARTH'S CURVATURE}

Because of the curvature of the earth $z_{v}$ is related to $\phi_{0}$ and $D$ by the relation

$$
\tan \phi_{0}=\frac{r \sin \theta}{z_{v}+r(1-\cos \theta)}
$$

where $r=$ radius of earth in kilometers

$\theta=222.4 / D$ degrees

$D=$ distance in kilometers.

Although Breit and Tuve's theorem is strictly true only for a plane earth, and there is some doubt as to its strict validity in the presence of the earth's magnetic field, experiment shows a remarkable agreement between skip distances observed and those calculated using this method. Because of penetration of the ionosphere the angle of incidence $\phi_{0}$ is not quite the same as that computed from the geometrical relation above, and the virtual heights used must be smaller than those measured, for the greater distances. Any difference in skip distance arising from this effect is probably less than the uncertainty in the characteristics of the ionosphere at latitudes and longitudes other than those where the ionosphere measurements are made.

\footnotetext{
4. F. Farmer and J. A. Ratcliffe, Wireless waves reflected from the ionosphere at oblique incidence. Proc. Phys. Soc. 48, 839 (1936).

W. Crone, K. Krüger, G. Goubau, and J. Zenneck, Echo measurements over long distances. Hochfrequenztechnik u. Elektroakustik 48,1 (1936).
} 


\section{APPROXIMATE METHOD OF CALCULATION}

For rapid approximate calculation, use may be made of the fact that the transmission curve for the ordinary ray is very nearly a secant-law curve, and of the fact that as the distance of transmission is increased, the separation of the ordinary and extraordinary limiting frequencies decreases rapidly. For this purpose, the frequency-virtual-height curve is plotted on semilogarithmic paper, the frequencies being plotted logarithmically. Transmission curves for various distances are plotted semilogarithmically on transparent paper as virtual heights vs. reciprocals of sec $\phi_{0}$. The reciprocals of sec $\phi_{0}$ are used, since it is necessary to divide the transmitted frequency by sec $\phi_{0}$ to obtain the

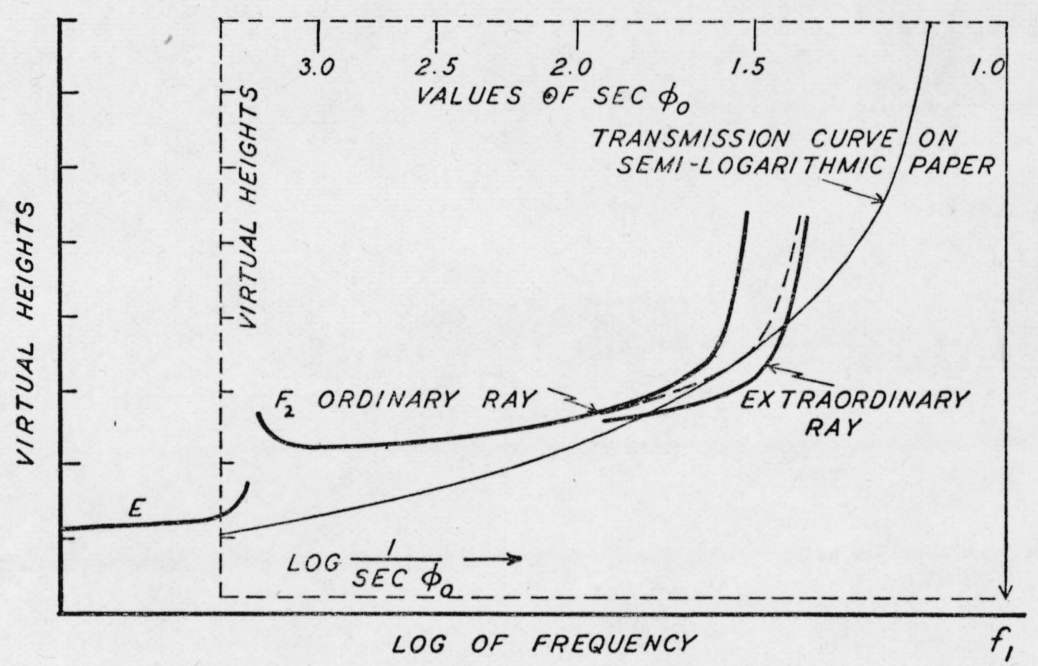

FIGURE 3.-Logarithmic transmission curve for rapid determination of limiting frequency

The transparent paper with the transmission curve on it is moved horizontally until the curve is just tangent to the frequency-virtual-height curve. The limiting frequency for the given distance is then read off opposite the line sec $\phi_{0}=1$. The dotted curve is drawn between the ordinary and extraordinary rays in order to take care of the decreasing separation of the rays at greater distances.

equivalent normal-incidence frequency. The transmission curves are then superimposed on the frequency-virtual-height curve for the ordinary component, as shown in figure 3, and moved until the transmission curve for the desired distance is just tangent to the frequencyvirtual-height curve. The frequency corresponding to sec $\phi_{0}=1$ (marked $f_{1}$ in fig. 3 ) is then the maximum usable frequency for that distance. For a closer approximation, the frequency-virtual-height curve may be corrected by drawing an estimated transition curve between the ordinary and extraordinary components, as shown by the thin dashed line in figure 3. This will give better values for short distances. This method is being used in preparing data for the weekly ionosphere broadcasts of the National Bureau of Standards.

A discussion of radio transmission and the ionosphere is being published in the June issue of the Journal of Research of the National Bureau of Standards and in the Proceedings of the Institute of Radio Engineers, and is available in the form of a Letter Circular of the National Bureau of Standards. 
94 Journal of Research of the National Bureau of Standards

A type of transmission curve based on the principles described above, was developed independently by L. V. Berkner, of the Department of Terrestrial Magnetism, Carnegie Institution of Washington, and described by him at the joint meeting of the IRE and URSI at Washington, on April 30, 1937.

Washington, May 28, 1937. 